\title{
On an extension of Hadamard fractional derivative
}

\author{
Muhammad Samraiz ${ }^{1}$, Erhan Set ${ }^{2}$, Muhammad Hasnain $^{1 *}$ and Gauhar Rahman ${ }^{3}$
}

\author{
"Correspondence: \\ rhasnainshahzad@gmail.com \\ 'Department of Mathematics, \\ University of Sargodha, Sargodha, \\ Pakistan \\ Full list of author information is \\ available at the end of the article
}

\begin{abstract}
In this paper, we introduce a new approach to the fractional derivation which generalizes the classical Hadamard fractional derivative. We prove some properties of this new approach and also establish some results by addressing some standard functions.

MSC: 26D15; 26D10;26A33

Keywords: Fractional calculus; Hadamard fractional derivative
\end{abstract}

\section{Introduction}

The subject of evaluating integrals and derivatives of any arbitrary real or complex order has gained considerable attention of mathematicians and scientists because of its exhibited applications in science, engineering and other related fields. The scientists working in the fields of inequalities and control theory found interesting outcomes when they apply the fractional calculus in their fields $[1,9,15,16]$. It was observed by several authors that fractional derivatives and integrals are more suitable for describing real materials. Some physical problems were also treated by using derivatives of non-integer orders. Nowadays, some of the application areas of fractional calculus include fluid flow, dynamical processes in self-similar, electrical networks, probability and statistics, control theory of dynamical systems, viscoelasticity, electrochemistry of corrosion, chemical physics etc. The generalization of fractional differential calculus operators were subjected to an intense debate in the last few years. For current progress on fractional calculus, the reader may also see $[5,13]$. There are different familiar forms of the fractional derivatives which have been studied broadly $[3,5,10,13,14]$. The first is the Riemann-Liouville fractional derivatives of order $\mu \in \mathbb{C}, \mathfrak{R}(\mu) \geq 0$, defined by

$$
\begin{aligned}
\left(D_{a+}^{\mu} f\right)(x) & :=\left(\frac{d}{d x}\right)^{m}\left(I_{a+}^{n-\mu} f\right)(x) \\
& =\frac{1}{\Gamma(m-\mu)}\left(\frac{d}{d x}\right)^{m} \int_{a}^{x} \frac{f(t)}{(x-t)^{\mu-m+1}} d t, \quad m=[\Re(\mu)]+1 ; x>a,
\end{aligned}
$$

(c) The Author(s) 2019. This article is distributed under the terms of the Creative Commons Attribution 4.0 International License (http://creativecommons.org/licenses/by/4.0/), which permits unrestricted use, distribution, and reproduction in any medium, provided you give appropriate credit to the original author(s) and the source, provide a link to the Creative Commons license, and indicate if changes were made. 
and

$$
\begin{aligned}
\left(D_{b-}^{\mu} f\right)(x) & :=\left(-\frac{d}{d x}\right)^{m}\left(I_{b-}^{m-\mu} f\right)(x) \\
& =\frac{1}{\Gamma(m-\mu)}\left(-\frac{d}{d x}\right)^{m} \int_{a}^{x} \frac{f(t)}{(t-x)^{\mu-m+1}} d t, \quad m=[\Re(\mu)]+1 ; x<b,
\end{aligned}
$$

respectively, where

$$
\left(I_{a+}^{\mu} f\right)(x)=\frac{1}{\Gamma(\mu)} \int_{a}^{x} \frac{f(t)}{(x-t)^{1-\mu}} d t
$$

and

$$
\left(I_{b-}^{\mu} f\right)(x)=\frac{1}{\Gamma(\mu)} \int_{x}^{b} \frac{f(t)}{(t-x)^{1-\mu}} d t
$$

are the left and right sided Riemann-Liouville fractional integrals.

Diaz et al. in [4] introduced the $k$-gamma function as follows.

Definition 1.1 Let $k>0$ and $\mathfrak{R}(\mu)>0$. The $k$-gamma function is given by the following integral:

$$
\Gamma_{k}(\mu)=\int_{0}^{\infty} t^{\mu-1} \exp \left(-\frac{t^{k}}{k}\right) d t .
$$

The fractional integrals introduced by Hadamard [8] are given in the next definition.

Definition 1.2 Let $(a, b)$ be a finite or infinite interval of the half-axis $\mathbb{R}^{+}$and let $\mathfrak{R}(\mu)>0$. We consider the left and right sided Hadamard fractional integrals of order $\mu \in \mathbb{C}, \mathfrak{R}(\mu)>$ 0 , defined as

$$
\left(\mathfrak{I}_{a^{+}}^{\mu} g\right)(x)=\frac{1}{\Gamma(\mu)} \int_{a}^{x}\left(\log \frac{x}{u}\right)^{\mu-1} \frac{g(u)}{u} d u, \quad a<x<b,
$$

and

$$
\left(\mathfrak{I}_{b^{-}}^{\mu} g\right)(x)=\frac{1}{\Gamma(\mu)} \int_{x}^{b}\left(\log \frac{u}{x}\right)^{\mu-1} \frac{g(u)}{u} d u, \quad a<x<b,
$$

respectively.

Hadamard fractional derivatives are introduced by using Hadamard fractional integrals as in the following.

Definition 1.3 ([8]) Let $\delta=x \mathfrak{D}\left(\mathfrak{D}=\frac{d}{d x}\right)$ be the delta derivative see [11, p. 2]. The left and right sided Hadamard fractional derivatives of order $\mu \in \mathbb{C}, m=[\Re(\mu)]+1$ with $\Re(\mu) \geq 0$ on $(a, b)$ are defined by

$$
\begin{aligned}
\left({ }^{H} \mathfrak{D}_{a+}^{\mu} f\right)(x) & :=(\delta)^{m}\left(\mathfrak{I}_{a+}^{m-\mu} f\right)(x) \\
& =\frac{(-1)^{m} \Gamma(\mu+1)}{B(\mu-m+1, m-\mu)} \int_{a}^{x}\left(\log \frac{x}{u}\right)^{-\mu-1} \frac{f(u)}{u} d u ; \quad a<x<b,
\end{aligned}
$$


and

$$
\begin{aligned}
\left({ }^{H} \mathfrak{D}_{b-}^{\mu} f\right)(x) & :=(-\delta)^{m}\left(\mathfrak{I}_{b-}^{m-\mu} f\right)(x) \\
& =\frac{(-1)^{m} \Gamma(\mu+1)}{B(\mu-m+1, m-\mu)} \int_{x}^{b}\left(\log \frac{u}{x}\right)^{-\mu-1} \frac{f(u)}{u} d u ; \quad a<x<b,
\end{aligned}
$$

respectively, where $\mathfrak{I}_{a+}^{\mu}$ and $\mathfrak{I}_{b-}^{\mu}$ are the left and right sided Hadamard fractional integrals.

The $k$ extension of Hadamard fractional integral introduced by Farid et al. is given in the following.

Definition 1.4 ([6]) Let $k, \mu$ and $a$ be positive real numbers with $u>a$, then the Hadamard $k$-fractional integral of order $\mu$ is defined as

$$
{ }_{H}^{k} \Im_{(a, u)}^{\mu} g(x)=\frac{1}{k \Gamma_{k}(\mu)} \int_{a}^{x}\left(\log \frac{x}{u}\right)^{\frac{\mu}{k}-1} \frac{g(u)}{u} d u .
$$

Now we give the definition of the Caputo-Hadamard fractional derivative presented in [2].

Definition 1.5 The fractional derivative ${ }_{a}^{\mathrm{CH}} D_{t}^{\mu}$ of order $0<\mu<1$ on $(a, b)$ is defined as:

$$
{ }_{a}^{\mathrm{CH}} D_{t}^{\mu} x(t)=\frac{t}{\Gamma(1-\mu)} \frac{d}{d t} \int_{a}^{t}\left(\log \frac{t}{\tau}\right)^{-\mu} \frac{x(\tau)-x(a)}{\tau} d \tau,
$$

which can be written as

$$
{ }_{a}^{\mathrm{CH}} D_{t}^{\mu} x(t)=\frac{1}{\Gamma(1-\mu)} \int_{a}^{t}\left(\log \frac{t}{\tau}\right)^{-\mu} x^{\prime}(\tau) d \tau .
$$

Diaz et al. introduced the following definition of the $k$-beta function and the Pochhammer $k$-symbol in [4].

Definition 1.6 Let $\mu$ be a complex number, $k$ be a real number and $m$ be a natural number. Then the Pochhammer $k$-symbol is given as

$$
(\mu)_{m, k}=(\mu)(\mu+k)(\mu+2 k) \cdots(\mu+(m-1) k) .
$$

The formula which relates the $k$-gamma function and the Pochhammer $k$-symbol is given as

$$
(\mu)_{m, k}=\frac{\Gamma_{k}(\mu+m k)}{\Gamma_{k}(\mu)} .
$$

Definition 1.7 The $k$-beta function $B_{k}(\mu, v)$ is defined by the formula

$$
B_{k}(\mu, v)=\frac{1}{k} \int_{0}^{1} \tau^{\frac{\mu}{k}-1}(1-\tau)^{\frac{\nu}{k}-1} d \tau, \quad \mathfrak{R}(\mu)>0, \mathfrak{R}(\nu)>0
$$


and the identity which relates it with the $\Gamma_{k}$ function is

$$
B_{k}(\mu, v)=\frac{\Gamma_{k}(\mu) \Gamma_{k}(v)}{\Gamma_{k}(\mu+v)} .
$$

The following definition of the incomplete gamma function is given in [7, p. 899].

Definition 1.8 The incomplete gamma function $\gamma(\mu, x)$ is defined by the formula

$$
\gamma(\mu, x)=\int_{0}^{x} \exp (-t) t^{\mu-1} d t, \quad \mathfrak{R}(\mu)>0 .
$$

In [12, p. 664], Fubini's theorem is stated as follows.

Definition 1.9 Let $\left(\Delta, \Sigma_{1}, \theta\right),\left(\Lambda, \Sigma_{2}, \phi\right)$ be two measure spaces. Suppose that $\theta$ and $\phi$ are complete and $\mu: \Delta \times \Lambda \rightarrow[-\infty, \infty]$ be $(\theta \times \phi)$-integrable. Then, for $\theta$-a.e. $t \in \Delta$, the function $\alpha(t, \cdot)$ is $\phi$-integrable and the function $\int_{\Lambda} \mu(\cdot, \tau) d \phi(\tau)$ is $\theta$-integrable. Similarly, for $\phi$-a.e. $\tau \in \Omega_{2}$ the function $\alpha(\cdot, \tau)$ is $\theta$-integrable and the function $\int_{\Delta} \alpha(t, \cdot) d \theta(t)$ is $\phi$ integrable. Moreover,

$$
\int_{\Delta}\left(\int_{\Lambda} \alpha(t, \tau) d \phi(\tau)\right) d \theta(t)=\int_{\Lambda}\left(\int_{\Delta} \alpha(t, \tau) d \theta(t)\right) d \phi(\tau)
$$

Tonelli's theorem is given by the following [12, p. 664].

Definition 1.10 Let $\left(\Delta, \Sigma_{1}, \theta\right),\left(\Lambda, \Sigma_{2}, \phi\right)$ be two measure spaces and suppose that $\theta$ and $\phi$ are complete and $\sigma$-finite and let $\alpha: \Delta \times \Lambda \rightarrow[0, \infty]$ be $\left(\Sigma_{1} \times \Sigma_{1}\right)$-measurable function. Then, for $\theta$-a.e. $t \in \Delta$, the function $\alpha(t, \cdot)$ measurable and the function $\int_{\Lambda} \alpha(\cdot, \tau) d \phi(\tau)$ is measurable. Similarly, for $\phi$-a.e. $\tau \in \Lambda$ the function $\alpha(\cdot, \tau)$ is measurable and the function $\int_{\Delta} \alpha(t, \cdot) d \theta(t)$ is measurable. Moreover,

$$
\int_{\Delta}\left(\int_{\Lambda} \alpha(t, \tau) d \phi(\tau)\right) d \theta(t)=\int_{\Lambda}\left(\int_{\Delta} \alpha(t, \tau) d \theta(t)\right) d \phi(\tau) .
$$

\section{Main results}

In continuation of the extensions of different fractional integrals and derivatives, we are going to define the following extension of the Hadamard fractional derivative.

Definition 2.1 Let $(a, b)$ be a finite or infinite interval of half-axis $\mathbb{R}^{+}$and let $\mathfrak{R}(\alpha-m+k)>$ $0, \mathfrak{R}(m-\mu)>0, \mu \in \mathbb{C}$ with $\mathfrak{R}(\mu)>0$. Then the left and right sided extended Hadamard fractional derivatives of order $\mu$ are defined by

$$
\begin{aligned}
\left({ }^{H} \mathfrak{D}_{a^{+}}^{\mu, k} f\right)(x)= & \delta^{m}\left({ }_{H} \mathfrak{I}_{a^{+}}^{m-\mu, k} f\right)(x) \\
= & \frac{(-1)^{m} \Gamma_{k}(\mu-m+k+n k)}{k^{m+1} B_{k}(\mu-m+k, m-\mu)} \int_{a}^{x}\left(\log \frac{x}{u}\right)^{\frac{m-\mu}{k}-m-1} \frac{f(u)}{u} d u \\
& (a<x<b)
\end{aligned}
$$


and

$$
\begin{aligned}
\left({ }^{H} \mathfrak{D}_{b^{-}}^{\mu, k} f\right)(x)= & (-\delta)^{m}\left(\begin{array}{l}
k \\
H
\end{array} \mathfrak{I}_{b^{-}}^{m-\mu} f\right)(x) \\
= & \frac{(-1)^{m} \Gamma_{k}(\mu-m+k+n k)}{k^{m+1} B_{k}(\mu-m+k, m-\mu)} \int_{x}^{b}\left(\log \frac{u}{x}\right)^{\frac{m-\mu}{k}-m-1} \frac{f(u)}{u} d u \\
& (a<x<b),
\end{aligned}
$$

respectively, where $m=[\Re(\mu)]+1, k>0$ and $\delta=x \frac{d}{d x}$ (delta derivative).

Remark 2.2 By setting $k=1$ in (2.1), we get

$$
\begin{aligned}
\left({ }^{H}\right. & \left.\mathfrak{D}_{a^{+}}^{\mu} f\right)(x) \\
& =\frac{(-1)^{m} \Gamma(\mu-m+1+m)}{1^{m+1} B(\mu-m+1, m-\mu)} \int_{a}^{x}\left(\log \frac{x}{u}\right)^{m-\mu-m-1} \frac{f(u)}{u} d u \\
& =\frac{(-1)^{m} \Gamma(\mu+1)}{B(\mu-m+1, m-\mu)} \int_{a}^{x}\left(\log \frac{x}{u}\right)^{-\mu-1} \frac{f(u)}{u} d u,
\end{aligned}
$$

which is the left side classical Hadamard fractional derivative.

Similarly, on substituting $k=1$ in (2.2) we can obtain the right sided classical Hadamard fractional derivative.

The following result proves the boundedness of the operator (2.1).

Theorem 2.3 Let $k>0, \mathfrak{R}\left(\frac{p(m-\mu)-k(m p+p-1)}{k}\right)>0$ and ${ }^{H} \mathfrak{D}_{a^{+}}^{\mu, k} f$ be the extended Hadamard fractional derivative of order $\mu \in \mathbb{C}$ such that $\mathfrak{R}(\mu)>0$ with $m=[\Re(\mu)]+1$ defined on $(a, b)$. Then the following result holds:

$$
\left|\left({ }^{H} \mathfrak{D}_{a^{+}}^{\mu, k} f\right)(x)\right| \leq C\|f(u)\|_{q}
$$

where $C=\frac{\Gamma_{k}(\mu-m+k+m k) x^{\frac{1-p}{p}}(1-p)^{1+m-\frac{(m-\mu)}{k}}}{k^{m+1} B_{k}(\mu-m+k, m-\mu)}\left(\Upsilon\left(\frac{p(m-\mu)-k(m p+p-1)}{k}, \log \frac{x}{a}\right)\right)^{\frac{1}{p}}$ and $\Upsilon(\mu, x)$ is the incomplete gamma function given by Definition 1.8 .

Proof

$$
\left|\left({ }^{H} \mathfrak{D}_{a^{+}}^{\mu, k} f\right)(x)\right| \leq \frac{\Gamma_{k}(\mu-m+k+m k)}{k^{m+1} B_{k}(\mu-m+k, m-\mu)} \int_{a}^{x}\left(\log \frac{x}{u}\right)^{\frac{m-\mu}{k}-m-1} \frac{|f(u)|}{u} d u .
$$

By applying Hölder's inequality, we obtain

$$
\begin{aligned}
\left|\left({ }^{H} \mathfrak{D}_{a^{+}}^{\mu, k} f\right)(x)\right| \leq & \frac{\Gamma_{k}(\mu-m+k+m k)}{k^{m+1} B_{k}(\mu-m+k, m-\mu)} \\
& \times\left(\int_{a}^{x}\left(\log \frac{x}{u}\right)^{\frac{p(m-\mu)}{k}-m p-p} \frac{1}{u^{p}} d u\right)^{\frac{1}{p}}\|f(u)\|_{L_{q}(a, x)^{*}}
\end{aligned}
$$


Substituting $\log \frac{x}{u}=z$, using the incomplete gamma function and after simple calculation, we get

$$
\begin{aligned}
\left|\left({ }^{H} \mathfrak{D}_{a^{+}}^{\mu, k} f\right)(x)\right| \leq & \frac{\Gamma_{k}(\mu-m+k+m k) x^{\frac{1-p}{p}}(1-p)^{1+m-\frac{(m-\mu)}{k}}}{k^{m+1} B_{k}(\mu-m+k, m-\mu)} \\
& \times\left(\Upsilon\left(\frac{p(m-\mu)-k(m p+p-1)}{k}, \log \frac{x}{a}\right)\right)^{\frac{1}{p}}\|f(u)\|_{L_{q}(a, x)} .
\end{aligned}
$$

The next theorem proves that the derivative defined by (2.1) is well defined.

Theorem 2.4 Let $f \in L_{1}\left[u^{\prime}, v^{\prime}\right], k>0$ and $\mathfrak{R}(\mu)>0$, then the derivative ${ }^{H} \mathfrak{D}_{a+}^{\mu, k} f$ of order $\mu \in \mathbb{C}$ with $m=[\Re(\mu)]+1$ exists for every $x \in\left(u^{\prime}, v^{\prime}\right)$.

Proof Let $\varrho:=\left[u^{\prime}, v^{\prime}\right] \times\left[u^{\prime}, v^{\prime}\right]$ and $\mathfrak{K}: \varrho \rightarrow \mathbb{R}$,

$$
\begin{aligned}
& \mathfrak{K}_{+}(x, y)= \begin{cases}\frac{(-1)^{m} \Gamma_{k}(\mu-m+k+m k)}{k^{m+1} B_{k}(\mu-m+k, m-\mu)}\left(\log \frac{x}{y}\right)^{\frac{m-\mu}{k}-m-1} \frac{1}{y}, & u^{\prime} \leq y \leq x \leq v^{\prime} ; \\
0, & u^{\prime} \leq x \leq y \leq v^{\prime},\end{cases} \\
& \mathfrak{K}_{-}(x, y)= \begin{cases}\frac{(-1)^{m} \Gamma_{k}(\mu-m+k+m k)}{k^{m+1} B_{k}(\mu-m+k, m-\mu)}\left(\log \frac{y}{x}\right)^{\frac{m-\mu}{k}-m-1} \frac{1}{y}, & u^{\prime} \leq x \leq y \leq v^{\prime} ; \\
0, & u^{\prime} \leq y \leq x \leq v^{\prime} .\end{cases}
\end{aligned}
$$

Since $\mathfrak{K}_{+}(x, y)$ is measurable on $\varrho$, we have

$$
\int_{u^{\prime}}^{v^{\prime}} \mathfrak{K}(x, y) d y=\frac{(-1)^{m+1} \Gamma_{k}(\mu-m+k+m k)}{k^{m+1} B_{k}(\mu-m+k, m-\mu)}\left(\log \frac{x}{u^{\prime}}\right)^{\frac{m-\mu}{k}-m} .
$$

By integrating repeatedly, we obtain

$$
\begin{aligned}
& \int_{u^{\prime}}^{v^{\prime}}\left(\int_{u^{\prime}}^{v^{\prime}} \mathfrak{K}(x, y)|f(x)| d y\right) d x \\
& \quad=\int_{u^{\prime}}^{v^{\prime}}|f(x)|\left(\int_{u^{\prime}}^{v^{\prime}} \mathfrak{K}(x, y) d y\right) d x \\
& \leq \frac{(-1)^{m+1} \Gamma_{k}(\mu-m+k+m k)}{k^{m} B_{k}(\mu-m+k, m-\mu)} \int_{u^{\prime}}^{v^{\prime}}|f(x)|\left(\log \frac{v^{\prime}}{u^{\prime}}\right)^{\frac{m-\mu}{k}-m} d x \\
& =\frac{(-1)^{m+1} \Gamma_{k}(\mu-m+k+m k)\left(\log \frac{v^{\prime}}{u^{\prime}}\right)^{\frac{m-\mu}{k}-m}}{k^{m} B_{k}(\mu-m+k, m-\mu)}\|f(x)\|_{L_{1}\left[u^{\prime}, v^{\prime}\right]}<\infty .
\end{aligned}
$$

Therefore, by applying Tonelli's theorem the function $\mathfrak{H}: \varrho \rightarrow R$, where $\mathfrak{H}(x, y):=$ $\mathfrak{K}(x, y) f(x)$ is integrable over $\varrho$. Hence $\int_{u^{\prime}}^{v^{\prime}} \mathfrak{K}(x, y) f(x) d x$ in integrable on $\left[u^{\prime}, v^{\prime}\right]$, as a function of $y \in\left[u^{\prime}, v^{\prime}\right]$ by using Fubini's theorem, i.e., $\left({ }^{H} \mathfrak{D}_{a^{+}}^{\mu, k} f\right)(x)$ exists.

Theorem 2.5 Let $k>0$ and $\mathfrak{R}(\mu), \mathfrak{R}(\beta)$ be positive with $n=[\Re(\mu)]+1$, then

$$
{ }^{H} \mathfrak{D}_{a+}^{\mu, k}\left({ }_{H}^{k} \mathfrak{I}_{a+}^{v} f(x)\right)=\left({ }^{H} \mathfrak{D}_{a+}^{\mu-v, k} f\right)(x) .
$$


Proof By applying Definition 2.1, we have

$$
\left.H_{\mathfrak{D}_{a+}^{\mu, k}}^{\mu, k}{ }_{H}^{k} \mathfrak{I}_{a+}^{v} f(x)\right)=\delta_{H}^{m k} \mathfrak{I}_{a+}^{m-\mu}\left({ }_{H}^{k} \mathfrak{I}_{a+}^{v} f(x)\right)
$$

Now by using Definition 1.4 and Fubini's formula, we get

$$
\begin{aligned}
{ }_{H}^{k} \mathfrak{I}_{a+}^{m-\mu}\left({ }_{H}^{k} \mathfrak{I}_{a+}^{v} f(x)\right) \\
=\frac{1}{k^{2} \Gamma_{k}(m-\mu) \Gamma_{k}(v)} \\
\quad \times \int_{a}^{x}\left[\int_{u}^{x}\left(\log \frac{x}{\tau}\right)^{\frac{m-\mu}{k}-1}\left(\log \frac{\tau}{u}\right)^{\frac{v}{k}-1} \frac{d \tau}{\tau}\right] \frac{f(u)}{u} d u \\
=\frac{1}{k^{2} \Gamma_{k}(m-\mu) \Gamma_{k}(v)} \\
\quad \times \int_{a}^{x}\left[\int_{u}^{x}\left(\log \frac{x}{u}\right)^{\frac{m-\mu}{k}-1}\left(1-\frac{\log \frac{\tau}{u}}{\log \frac{x}{u}}\right)^{\frac{m-\mu}{k}-1}\left(\log \frac{\tau}{u}\right)^{\frac{v}{k}-1} \frac{d \tau}{\tau}\right] \frac{f(u)}{u} d u .
\end{aligned}
$$

Substituting $\log \frac{\tau}{u}=s \log \frac{x}{u}$, we get

$$
\begin{aligned}
{ }_{H} \mathfrak{I}_{a+}^{m-\mu, k}\left({ }_{H} \mathfrak{I}_{a+}^{v, k} f(x)\right) \\
=\frac{1}{k^{2} \Gamma_{k}(m-\mu) \Gamma_{k}(v)} \\
\quad \times \int_{a}^{x} \frac{f(u)}{u}\left[\int_{0}^{1}\left(\log \frac{x}{u}\right)^{\frac{m-\mu+v}{k}-1}(s)^{\frac{v}{k}-1}(1-s)^{\frac{m-\mu}{k}-1} d s\right] d u .
\end{aligned}
$$

By using the $k$-beta function, its property and Definition 1.4, we obtain

$$
\begin{aligned}
{ }_{H} \mathfrak{I}_{a+}^{m-\mu, k}\left({ }_{H} \mathfrak{I}_{a+}^{v, k} f(x)\right) \\
\quad=\frac{B_{k}(\nu, m-\mu)}{k \Gamma_{k}(m-\mu) \Gamma_{k}(v)} \int_{a}^{x}\left(\log \frac{x}{u}\right)^{\frac{m-\mu+v}{k}-1} \frac{f(u)}{u} d u \\
\quad=\frac{1}{k \Gamma_{k}(m-\mu+v)} \int_{a}^{x}\left(\log \frac{x}{u}\right)^{\frac{m-\mu+v}{k}-1} \frac{f(u)}{u} d u \\
=\left(\begin{array}{l}
k \\
H
\end{array} \mathfrak{I}_{a+}^{m-(\mu-v)} f\right)(x) .
\end{aligned}
$$

By using Definition 2.1, we get

$$
{ }^{H} \mathfrak{D}_{a+}^{\mu, k}\left({ }_{H} \mathfrak{I}_{a+}^{v, k} f(x)\right)={ }^{H} \mathfrak{D}_{a+}^{\mu-v, k} f(x) .
$$

This completes the proof.

The following theorem proves the linearity of our operator.

Theorem 2.6 Let $a, b$ be scalars and all the assumptions of Theorem 2.5 be true. Then the following result holds:

$$
{ }^{H} \mathfrak{D}_{a}^{\mu, k}(\mathrm{a} f(x)+\mathrm{b} g(x))=\mathrm{a}\left({ }^{H} \mathfrak{D}_{a}^{\mu, k}\right) f(x)+\mathrm{b}\left({ }^{H} \mathfrak{D}_{a}^{\mu, k}\right) g(x) .
$$


Proof By applying Definition 2.1, we obtain

$$
{ }^{H} \mathfrak{D}_{a}^{\mu, k}(\mathrm{a} f(x)+\mathrm{b} g(x))=\frac{1}{k \Gamma_{k}(m-\mu)}\left(x \frac{d}{d x}\right)^{m} \int_{a}^{x}\left(\log \frac{x}{u}\right)^{\frac{m-\mu}{k}-1} \frac{(\mathrm{a} f(u)+\mathrm{b} g(u))}{u} d u .
$$

Now by using the linearity of integration, we have

$$
\begin{aligned}
H_{\mathfrak{D}_{a}}^{\mu, k}(\mathrm{a} f(x)+\mathrm{b} g(x))= & \frac{\mathrm{a}}{k \Gamma_{k}(m-\mu)}\left(x \frac{d}{d x}\right)^{m} \int_{a}^{x}\left(\log \frac{x}{u}\right)^{\frac{m-\mu}{k}-1} \frac{f(u)}{u} d u \\
& +\frac{\mathrm{b}}{k \Gamma_{k}(m-\mu)}\left(x \frac{d}{d x}\right)^{m} \int_{a}^{x}\left(\log \frac{x}{u}\right)^{\frac{m-\mu}{k}-1} \frac{g(u)}{u} d u
\end{aligned}
$$

which gives the required result.

Lemma 2.7 The extended Hadamard fractional derivative operator of any constant $C$ of order $\mu$ such that $\mathfrak{R}(\mu)>0$ with $m=[\Re(\mu)]+1$, is

$$
\left({ }^{H} \mathfrak{D}_{a^{+}}^{\mu, k}\right) C=\frac{(-1)^{m+1} C \Gamma_{k}(\mu-m+m k)}{k^{m} B_{k}(\mu-m+k, m-\mu)}\left(\log \frac{x}{a}\right)^{\frac{m-\mu}{k}-m},
$$

i.e., the extended Hadamard fractional derivative of constant function does not vanish.

Proof

$$
\left({ }^{H} \mathfrak{D}_{a^{+}}^{\mu, k}\right) C=\frac{(-1)^{m} C \Gamma_{k}(\mu-m+k+m k)}{k^{m+1} B_{k}(\mu-m+k, m-\mu)} \int_{a}^{x}\left(\log \frac{x}{u}\right)^{\frac{m-\mu}{k}-m-1} \frac{1}{u} d u .
$$

By integrating the right hand side, we get (2.6).

Lemma 2.8 Let $\mathfrak{R}(\mu)>0, \mathfrak{R}\left(\frac{m-\mu-n k}{k}\right)>0$, then extended Hadamard fractional derivative of the function $f(x)=x^{2}+2 x$ of order $\mu$ with $m=[\mathfrak{R}(\mu)]+1$, is given by

$$
\begin{aligned}
\left({ }^{H} \mathfrak{D}_{a^{+}}^{\mu, k}\right)\left(x^{2}+2 x\right) & \\
\quad= & \frac{(-1)^{m} 2 x \Gamma_{k}(\mu-m+k+m k) \Upsilon\left(\frac{m-\mu-m k}{k}, \log \frac{x}{a}\right)}{k^{m+1} B_{k}(\mu-m+k, m-\mu)}\left[2^{\frac{m k-m+\mu}{k}} x+1\right] .
\end{aligned}
$$

Proof By applying the definition of left sided extended Hadamard fractional derivative, we have

$$
\left({ }^{H} \mathfrak{D}_{a^{+}}^{\mu, k}\right)\left(x^{2}+2 x\right)=\frac{(-1)^{m} \Gamma_{k}(\mu-m+k+m k)}{k^{m+1} B_{k}(\mu-m+k, m-\mu)} \int_{a}^{x}\left(\log \frac{x}{u}\right)^{\frac{m-\mu}{k}-m-1}(u+2) d u .
$$

Substituting $\log \frac{x}{u}=z$ and using the definition of incomplete gamma function, we obtain Eq. (2.7).

Theorem 2.9 Let $\mathfrak{R}(\mu)>0, \mathfrak{R}(v)>0$, with $n=[\Re(\mu)]+1$ and $m=[\Re(v)]+1$ such that $n$ and $m$ are even. Let $f$ and $g$ be synchronous on $[0, \infty)$. Then for all $u>a \geq 0$ the following 
inequalities hold for extended Hadamard fractional derivative:

$$
{ }^{H} \mathfrak{D}_{a+}^{\mu, k} f g(u) \geq \frac{1}{H \mathfrak{D}_{a+}^{\mu, k}(1)}{ }^{H} \mathfrak{D}_{a+}^{\mu, k} f(u)^{H} \mathfrak{D}_{a+}^{\mu, k} g(u)
$$

and

$$
\begin{aligned}
{ }^{H} & \mathfrak{D}_{a+}^{\mu, k} f g(u)^{H} \mathfrak{D}_{a+}^{v, k}(1)+{ }^{H} \mathfrak{D}_{a+}^{v, k} f g(u)^{H} \mathfrak{D}_{a+}^{\mu, k}(1) \\
& \geq{ }^{H} \mathfrak{D}_{a+}^{\mu, k} f(u)^{H} \mathfrak{D}_{a+}^{v, k} g(u)+{ }^{H} \mathfrak{D}_{a+}^{\mu, k} g(u)^{H} \mathfrak{D}_{a_{+}}^{v} f(u) .
\end{aligned}
$$

Proof For the synchronous functions $f$ and $g$ on $[0, \infty)$, we have

$$
(f(t)-f(\tau))(g(t)-g(\tau)) \geq 0 \quad \forall t, \tau \geq 0 .
$$

Therefore

$$
f(t) g(t)+f(\tau) g(\tau) \geq f(t) g(\tau)+f(\tau) g(t) .
$$

Multiplying both sides of (2.10) with $\frac{\Gamma_{k}(\mu-n+k+n k)}{k^{n+1} B_{k}(\mu-n+k, n-\mu)}\left(\log \frac{u}{t}\right)^{\frac{n-\mu}{k}-n-1} \frac{1}{t}$, then integrating the resulting inequality with respect to $t$ over $(a, u)$, we obtain

$$
\begin{gathered}
\frac{\Gamma_{k}(\mu-n+k+n k)}{k^{n+1} B_{k}(\mu-n+k, n-\mu)} \int_{a}^{u}\left(\log \frac{u}{t}\right)^{\frac{n-\mu}{k}-n-1} \frac{1}{t} f(t) g(t) d t \\
\quad+\frac{\Gamma_{k}(\mu-n+k+n k)}{k^{n+1} B_{k}(\mu-n+k, n-\mu)} \int_{a}^{u}\left(\log \frac{u}{t}\right)^{\frac{n-\mu}{k}-n-1} \frac{1}{t} f(\tau) g(\tau) d t \\
\geq \frac{\Gamma_{k}(\mu-n+k+n k)}{k^{n+1} B_{k}(\mu-n+k, n-\mu)} \int_{a}^{u}\left(\log \frac{u}{t}\right)^{\frac{n-\mu}{k}-n-1} \frac{1}{t} f(t) g(\tau) d t \\
\quad+\frac{\Gamma_{k}(\mu-n+k+n k)}{k^{n+1} B_{k}(\mu-n+k, n-\mu)} \int_{a}^{u}\left(\log \frac{u}{t}\right)^{\frac{n-\mu}{k}-n-1} \frac{1}{t} f(\tau) g(t) d t, \\
{ }_{\mathfrak{D}_{a+}^{\mu, k}} f g(u)+f(\tau) g(\tau)^{H} \mathfrak{D}_{a+}^{\mu, k}(1) \geq g(\tau)^{H} \mathfrak{D}_{a+}^{\mu, k} f(u)+f(\tau)^{H} \mathfrak{D}_{a+}^{\mu, k} g(u) .
\end{gathered}
$$

By multiplying $\frac{\Gamma_{k}(\mu-n+k+n k)}{k^{n+1} B_{k}(\mu-n+k, n-\mu)}\left(\log \frac{u}{\tau}\right)^{\frac{n-\mu}{k}-n-1} \frac{1}{\tau}$ with both sides of (2.11) and then integrating the obtained inequality with respect to $\tau$ over $(a, u)$, we obtain

$$
\begin{aligned}
{ }^{H} \mathfrak{D}_{a+}^{\mu, k} f g(u) \frac{\Gamma_{k}(\mu-n+k+n k)}{k^{n+1} B_{k}(\mu-n+k, n-\mu)} \int_{a}^{u}\left(\log \frac{u}{\tau}\right)^{\frac{n-\mu}{k}-n-1} \frac{1}{\tau} d \tau \\
\quad+{ }^{H} \mathfrak{D}_{a+}^{\mu, k}(1) \frac{\Gamma_{k}(\mu-n+k+n k)}{k^{n+1} B_{k}(\mu-n+k, n-\mu)} \int_{a}^{u}\left(\log \frac{u}{\tau}\right)^{\frac{n-\mu}{k}-n-1} \frac{1}{\tau} f(\tau) g(\tau) d \tau \\
\geq{ }^{H} \mathfrak{D}_{a+}^{\mu, k} f(u) \frac{\Gamma_{k}(\mu-n+k+n k)}{k^{n+1} B_{k}(\mu-n+k, n-\mu)} \int_{a}^{u}\left(\log \frac{u}{\tau}\right)^{\frac{n-\mu}{k}-n-1} \frac{1}{\tau} g(\tau) d \tau \\
+{ }^{H} \mathfrak{D}_{a^{+}}^{\mu, k} g(u) \frac{\Gamma_{k}(\mu-n+k+n k)}{k^{n+1} B_{k}(\mu-n+k, n-\mu)} \int_{a}^{u}\left(\log \frac{u}{\tau}\right)^{\frac{n-\mu}{k}-n-1} \frac{1}{\tau} f(\tau) d \tau,
\end{aligned}
$$

which proves the result (2.8). 
By multiplying $\frac{\Gamma_{k}(v-m+k+m k)}{k^{m+1} B_{k}(v-m+k, m-\mu)}\left(\log \frac{u}{\tau}\right)^{\frac{m-v}{k}-m-1} \frac{1}{\tau}$ with both sides of (2.11) and then integrating the obtained inequality with respect to $\tau$ over $(a, u)$, we get

$$
\begin{aligned}
& H \mathfrak{D}_{a+}^{\mu, k} f g(u) \frac{\Gamma_{k}(v-m+k+m k)}{k^{m+1} B_{k}(v-m+k, m-\mu)} \int_{a}^{u}\left(\log \frac{u}{\tau}\right)^{\frac{m-v}{k}-m-1} \frac{1}{\tau} d y \\
& \quad+{ }^{H} \mathfrak{D}_{a+}^{\mu, k}(1) \frac{\Gamma_{k}(v-m+k+m k)}{k^{m+1} B_{k}(v-m+k, m-\mu)} \int_{a}^{u}\left(\log \frac{u}{\tau}\right)^{\frac{m-v}{k}-m-1} \frac{1}{\tau} f(\tau) g(\tau) d y \\
& \geq{ }^{H} \mathfrak{D}_{a+}^{\mu, k} f(u) \frac{\Gamma_{k}(v-m+k+m k)}{k^{m+1} B_{k}(v-m+k, m-\mu)} \int_{a}^{u}\left(\log \frac{u}{\tau}\right)^{\frac{m-v}{k}-m-1} \frac{1}{\tau} g(\tau) d y \\
& \quad+{ }^{H} \mathfrak{D}_{a+}^{\mu, k} g(u) \frac{\Gamma_{k}(v-m+k+m k)}{k^{m+1} B_{k}(v-m+k, m-\mu)} \int_{a}^{u}\left(\log \frac{u}{\tau}\right)^{\frac{m-v}{k}-m-1} \frac{1}{\tau} f(\tau) d y .
\end{aligned}
$$

Hence the result (2.9) is proved.

Corollary 2.10 Let all the assumptions of Theorem 2.9 hold with $m$ and $n$ odd. Then the following inequalities hold:

$$
{ }^{H} \mathfrak{D}_{a+}^{\mu, k} f g(u) \leq{\frac{1}{H \mathfrak{D}_{a+}^{\mu, k} 1}}^{H} \mathfrak{D}_{a+}^{\mu, k} f(u)^{H} \mathfrak{D}_{a+}^{\mu, k} g(u)
$$

and

$$
\begin{array}{rl}
{ }^{H} \mathfrak{D}_{a_{+}}^{\mu, k} & f g(u)^{H} \mathfrak{D}_{a+}^{v, k}(1)+{ }^{H} \mathfrak{D}_{a+}^{v, k} f g(u)^{H} \mathfrak{D}_{a+}^{\mu, k}(1) \\
& \leq{ }^{H} \mathfrak{D}_{a+}^{\mu, k} f(u)^{H} \mathfrak{D}_{a_{+}}^{v, k} g(u)+{ }^{H} \mathfrak{D}_{a+}^{\mu, k} g(u)^{H} \mathfrak{D}_{a_{+}}^{v} f(u) .
\end{array}
$$

To establish the next results, we need the following lemma.

Lemma 2.11 Let $\mathfrak{R}(\mu)$ and $\mathfrak{R}(\nu)$ be positive with $m=[\Re(\mu)]+1$ and $0<a<b<\infty$, then

$$
\left(\mathfrak{I}_{a^{+}}^{m-\mu, k}\left(\log \frac{x}{a}\right)^{\frac{v}{k}-1}\right)=\frac{\Gamma_{k}(v)}{\Gamma_{k}(m-\mu+v)}\left(\log \frac{x}{a}\right)^{\frac{m-\mu+v}{k}-1} .
$$

Proof We have

$$
\begin{aligned}
& \left(\mathfrak{I}_{a^{+}}^{m-\mu, k}\left(\log \frac{x}{a}\right)^{\frac{v}{k}-1}\right) \\
& =\frac{1}{k \Gamma_{k}(m-\mu)} \int_{a}^{x}\left(\log \frac{x}{u}\right)^{\frac{m-\mu}{k}-1}\left(\log \frac{u}{a}\right)^{\frac{v}{k}-1} \frac{d u}{u} \\
& =\frac{1}{k \Gamma_{k}(m-\mu)} \int_{a}^{x}\left(\log \frac{x}{a}\right)^{\frac{m-\mu}{k}-1}\left(1-\frac{\log \frac{u}{a}}{\log \frac{x}{a}}\right)^{\frac{m-\mu}{k}-1}\left(\log \frac{u}{a}\right)^{\frac{v}{k}-1} \frac{d u}{u}
\end{aligned}
$$

Substituting $\log \frac{u}{a}=s \log \frac{x}{a}$, then using the definition of the $k$-beta function and its property,we obtain inequality (2.12).

Lemma 2.12 Let $\mathfrak{R}(\mu)$ and $\mathfrak{R}(v)$ be positive with $m=[\Re(\mu)]+1$ and $0<a<b<\infty$, then

$$
\left(H \mathfrak{D}_{a^{+}}^{\mu, k}\left(\log \frac{x}{a}\right)^{\frac{v}{k}-1}\right)=\frac{\Gamma_{k}(v) \Gamma_{k}(\mu-m-v+k+m k)}{k^{m} B_{k}(m-\mu+v, \mu-m-v+k)}\left(\log \frac{x}{a}\right)^{\frac{m-\mu+v}{k}-m-1} .
$$


Proof By applying Definition 2.1, we get

$$
\left({ }^{H} \mathfrak{D}_{a^{+}}^{\mu, k}\left(\log \frac{x}{a}\right)^{\frac{v}{k}-1}\right)=x^{m} \frac{d^{m}}{d x^{m}}\left(\mathfrak{I}_{a^{+}}^{m-\mu, k}\left(\log \frac{x}{a}\right)^{\frac{v}{k}-1}\right) .
$$

Now by using (2.12) and after a small calculation, we arrive at the required result.

Remark 2.13 In particular, if $v=1, k=1$ and $m-1<\mu<m$, we get the classical Hadamard fractional derivative of one which is also not equal to zero;

$$
\left({ }^{H} \mathfrak{D}_{a^{+}}^{\mu} 1\right)(x)=\frac{(-1)^{m} \Gamma(\mu)}{\Gamma(m-\mu+1) \Gamma(\mu-m)}\left(\log \frac{x}{a}\right)^{-\mu} .
$$

Remark 2.14 Let $\mathfrak{R}(m-\mu-m k)>0$ and $\Re(\mu-m+k+m k)>0$. Then, for $x>1$ and positive real numbers $k, \mu$ and $\nu$, we have

$$
\begin{aligned}
\left({ }^{H} \mathfrak{D}_{(1, x)}^{\mu, k}(\log x)^{\frac{v}{k}-1}\right)= & \frac{(-1)^{m} B_{k}(m-\mu-m k, v) \Gamma_{k}(\mu-m+k+m k)}{k^{m} B_{k}(\mu-m+k, m-\mu)} \\
& \times(\log (x))^{\frac{m-\mu+v}{k}-m-1} .
\end{aligned}
$$

Proof

$$
\begin{aligned}
\left({ }^{H} \mathfrak{D}_{(1, x)}^{\mu, k}(\log x)^{\frac{v}{k}-1}\right)= & \frac{(-1)^{m} \Gamma_{k}(\mu-m+k+m k)}{k^{m+1} B_{k}(\mu-m+k, m-\mu)} \\
& \times \int_{1}^{x}\left(\log \frac{x}{u}\right)^{\frac{m-\mu}{k}-m-1}(\log u)^{\frac{v}{k}-1} \frac{d u}{u} \\
= & \frac{(-1)^{m} \Gamma_{k}(\mu-m+k+m k)}{k^{m+1} B_{k}(\mu-m+k, m-\mu)} \\
& \times \int_{1}^{x}(\log x)^{\frac{m-\mu}{k}-m-1}\left(1-\frac{\log (u)}{\log (x)}\right)^{\frac{m-\mu}{k}-m-1}(\log u)^{\frac{v}{k}-1} \frac{d u}{u} .
\end{aligned}
$$

Substituting $\log (u)=s \log (x)$, we obtain

$$
\begin{aligned}
\left({ }^{H} \mathfrak{D}_{(1, x)}^{\mu, k}(\log x)^{\frac{v}{k}-1}\right)= & \frac{(-1)^{m} \Gamma_{k}(\mu-m+k+m k)(\log (x))^{\frac{m-\mu+v}{k}-m-1}}{k^{m+1} B_{k}(\mu-m+k, m-\mu)} \\
& \times \int_{0}^{1}(1-s)^{\frac{m-\mu}{k}-m-1}(s)^{\frac{v}{k}-1} d s .
\end{aligned}
$$

Now by using $k$-beta function and its property, we get (2.13).

Remark 2.15 Let $\mathfrak{R}(m-\mu-m k)>0$ and $\mathfrak{R}(\mu-m+k+m k)>0$. Then, for $u>1$ and positive real numbers $k$ and $\mu$, we get

$$
\left({ }^{H} \mathfrak{D}_{(1, x)}^{\mu, k}(\log (x))\right)=\frac{(-1)^{m} B_{k}(\mu-m+k+m k, m-\mu-m k)(\log (x))^{\frac{m-\mu}{k}-m+1}}{k^{m-1} B_{k}(\mu-m+k, m-\mu) \Gamma_{k}(m-\mu-m k+2 k)} .
$$


Proof By applying the definition of the extended Hadamard fractional derivative, we get

$$
\begin{aligned}
\left({ }^{H} \mathfrak{D}_{(1, x)}^{\mu, k}(\log (x))\right)= & \frac{(-1)^{m} \Gamma_{k}(\mu-m+k+m k)}{k^{m+1} B_{k}(\mu-m+k, m-\mu)} \\
& \times \int_{1}^{x}\left(\log \frac{x}{u}\right)^{\frac{m-\mu}{k}-m-1}(\log u) \frac{d u}{u} \\
= & \frac{(-1)^{m} \Gamma_{k}(\mu-m+k+m k)}{k^{m+1} B_{k}(\mu-m+k, m-\mu)} \\
& \times \int_{1}^{x}(\log x)^{\frac{m-\mu}{k}-m-1}\left(1-\frac{\log (u)}{\log (x)}\right)^{\frac{m-\mu}{k}-m-1}(\log u)^{\frac{2 k}{k}-1} \frac{d u}{u} .
\end{aligned}
$$

Substituting $\log (u)=s \log (x)$, we have

$$
\begin{aligned}
\left({ }^{H}\right. & \left.\mathfrak{D}_{(1, x)}^{\mu, k}(\log x)^{\frac{v}{k}-1}\right) \\
\quad & =\frac{(-1)^{m} \Gamma_{k}(\mu-m+k+m k)}{k^{m+1} B_{k}(\mu-m+k, m-\mu)}(\log x)^{\frac{m-\mu}{k}-m+1} \int_{0}^{1}(1-s)^{\frac{m-\mu}{k}-m-1}(s)^{\frac{2 k}{k}-1} d s .
\end{aligned}
$$

Now by using the $k$-beta function, its property and after simple calculation, we obtain (2.14).

Remark 2.16 Let $k$ and $\mu$ be positive real numbers. Then, for $x>1$ and $\gamma>-1$, we get

$$
\begin{aligned}
\left({ }^{H}\right. & \left.\mathfrak{D}_{(1, x)}^{\mu, k}(\log (x))^{\gamma}\right) \\
& =(-1)^{m} \frac{\left.B_{k}(\mu-m+k+m k, m-\mu-m k) \Gamma_{k}(\gamma+1) k\right)(\log (x))^{\frac{m-\mu}{k}}+\gamma-m}{k^{m} B_{k}(\mu-m+k, m-\mu) \Gamma_{k}(m-\mu+(\gamma-m+1) k)} .
\end{aligned}
$$

Proof

$$
\begin{aligned}
\left({ }^{H} \mathfrak{D}_{(1, x)}^{\mu, k}(\log (x))^{\gamma}\right)(u)= & \frac{(-1)^{m} \Gamma_{k}(\mu-m+k+m k)}{k^{m+1} B_{k}(\mu-m+k, m-\mu)} \\
& \times \int_{1}^{x}\left(\log \frac{x}{u}\right)^{\frac{m-\mu}{k}-m-1}(\log u)^{\gamma} \frac{d u}{u} \\
= & \frac{(-1)^{m} \Gamma_{k}(\mu-m+k+m k)}{k^{m+1} B_{k}(\mu-m+k, m-\mu)} \\
& \times \int_{1}^{x}(\log x)^{\frac{m-\mu}{k}-m-1}\left(1-\frac{\log (u)}{\log (x)}\right)^{\frac{m-\mu}{k}-m-1}(\log u)^{\frac{\gamma k}{k}+1-1} \frac{d u}{u} .
\end{aligned}
$$

Substituting $\log (u)=s \log (x)$, we have

$$
\begin{aligned}
\left({ }^{H} \mathfrak{D}_{(1, x)}^{\mu, k}(\log x)^{\frac{v}{k}-1}\right)(u) \\
=\frac{(-1)^{m} \Gamma_{k}(\mu-m+k+m k)}{k^{m+1} B_{k}(\mu-m+k, m-\mu)}(\log x)^{\frac{m-\mu}{k}+\gamma-m} \\
\quad \times \int_{0}^{1}(1-s)^{\frac{m-\mu-m k}{k}-1}(s)^{\frac{\gamma k+k}{k}-1} d s .
\end{aligned}
$$


Now by using the $k$-beta function, its property and after simple calculation, we obtain (2.14).

Lemma 2.17 Let $\mathfrak{R}(\mu)>0, \mathfrak{R}(\mu-m+k)>0$ and $\mathfrak{R}(\mu-m+m k)>0$. Then the fractional derivatives $D_{a^{+}}^{\mu, k} f$ and $D_{b^{-}}^{\mu, k} f$ exist on $(a, b]$ and $[a, b)$, respectively, and are represented in the forms

$$
\begin{aligned}
\left({ }^{H} \mathfrak{D}_{a^{+}}^{\mu, k} f\right)(x) & \\
= & \frac{(-1)^{m+1} \Gamma_{k}(\mu-m+m k) f(a)}{k^{m} B_{k}(\mu-m+k, m-\mu)}\left(\log \frac{x}{a}\right)^{\frac{m-\mu}{k}-m} \\
& \quad+\frac{(-1)^{m+1} \Gamma_{k}(\mu-m+m k)}{k^{m} B_{k}(\mu-m+k, m-\mu)} \int_{a}^{x}\left(\log \frac{x}{u}\right)^{\frac{m-\mu}{k}-m} f^{\prime}(u) d u
\end{aligned}
$$

and

$$
\begin{aligned}
\left({ }^{H} \mathfrak{D}_{b^{-}}^{\mu, k} f\right)(x) & \\
= & \frac{(-1)^{m} \Gamma_{k}(\mu-m+m k) f(b)}{k^{m} B_{k}(\mu-m+k, m-\mu)}\left(\log \frac{b}{x}\right)^{\frac{m-\mu}{k}-m} \\
& \quad-\frac{(-1)^{m+1} \Gamma_{k}(\mu-m+m k)}{k^{m} B_{k}(\mu-m+k, m-\mu)} \int_{x}^{b}\left(\log \frac{u}{x}\right)^{\frac{m-\mu}{k}-m} f^{\prime}(u) d u,
\end{aligned}
$$

respectively.

Proof By using the definitions given by (2.1), (2.2) and then integrating by parts, we can obtain (2.15) and (2.16).

Lemma 2.18 If $\mathfrak{R}(\mu-m+k)>0, \mathfrak{R}(\mu)>0$ with $m=[\Re(\mu)]+1, k>0$ and $f \in C[a, b]$, then

$$
\begin{aligned}
& \left({ }^{H} \mathfrak{D}_{a^{+}}^{\mu, k} f\right)(a)=\lim _{u \rightarrow a}\left({ }^{H} \mathfrak{D}_{a^{+}}^{\mu, k} f\right)(u)=0, \\
& \left({ }^{H} \mathfrak{D}_{b^{-}}^{\mu, k} f\right)(b)=\lim _{u \rightarrow b}\left({ }^{H} \mathfrak{D}_{b^{-}}^{\mu, k} f\right)(u)=0 .
\end{aligned}
$$

Proof

$$
\begin{aligned}
\left({ }^{H} \mathfrak{D}_{a^{+}}^{\mu, k} f\right)(x) & =\frac{(-1)^{m} \Gamma_{k}(\mu-m+k+m k)}{k^{m+1} B_{k}(\mu-m+k, m-\mu)} \int_{a}^{x}\left(\log \frac{x}{u}\right)^{\frac{m-\mu}{k}-m-1} \frac{f(u)}{u} d u \\
& =\frac{(-1)^{m-1}(m-\mu-m k) \Gamma_{k}(\mu-m+m k)}{k^{m+1} B_{k}(\mu-m+k, m-\mu)} \int_{a}^{x}\left(\log \frac{x}{u}\right)^{\frac{m-\mu}{k}-m-1} \frac{f(u)}{u} d u .
\end{aligned}
$$

Since $f \in C[a, b]$, there is a constant $C$ such that $|f(u)| \leq C$. Therefore,

$$
\begin{aligned}
\left|\left(D_{a^{+}}^{\mu, k} f\right)(u)\right| & \leq \frac{(m-\mu-m k) \Gamma_{k}(\mu-m+m k)}{k^{m+1} B_{k}(\mu-m+k, m-\mu)} \int_{a}^{u}\left(\log \frac{u}{x}\right)^{\frac{m-\mu}{k}-m-1}|f(x)| \frac{d x}{x} \\
& \leq \frac{C(m-\mu-m k) \Gamma_{k}(\mu-m+m k)}{k^{m+1} B_{k}(\mu-m+k, m-\mu)} \int_{a}^{u}\left(\log \frac{u}{x}\right)^{\frac{m-\mu}{k}-m-1} \frac{d x}{x}
\end{aligned}
$$




$$
\begin{aligned}
& \leq \frac{C \Gamma_{k}(\mu-m+m k)}{k^{m} B_{k}(\mu-m+k, m-\mu)}\left(\log \frac{u}{a}\right)^{\frac{m-\mu}{k}-m}, \\
\left({ }^{H} \mathfrak{D}_{a}^{\mu, k} f\right)(a) & =\lim _{u \rightarrow a}\left({ }^{H} \mathfrak{D}_{a^{+}}^{\mu, k} f\right)(u)=0 .
\end{aligned}
$$

In a similar manner, we prove the second part of the lemma.

Theorem 2.19 If ${ }^{H} \mathfrak{D}_{a+}^{\mu, k} f={ }^{H} \mathfrak{D}_{a+}^{\mu, k} g$, then $f=g$.

Proof Since

$$
\left({ }^{H} \mathfrak{D}_{a}^{\mu, k} f\right)(x)=\frac{(-1)^{m} \Gamma_{k}(\mu-m+k+m k)}{k^{m+1} B_{k}(\mu-m+k, m-\mu)} \int_{a}^{x}\left(\log \frac{x}{u}\right)^{\frac{m-\mu}{k}-m-1} \frac{f(u)}{u} d u
$$

and ${ }^{H} \mathfrak{D}_{a+}^{\mu, k} f={ }^{H} \mathfrak{D}_{a+}^{\mu, k} g$, due to linearity of the integral, we can write

$$
\begin{aligned}
& \int_{a}^{x} \frac{1}{u}\left(\log \frac{x}{u}\right)^{\frac{m-\mu}{k}-m-1}(f(u)-g(u)) d u=0, \\
& f(u)-g(u)=0
\end{aligned}
$$

which implies $f(u)=g(u)$.

\section{Conclusion}

The article includes a new approach to the fractional derivation which generalizes the classical Hadamard fractional derivative. The boundedness and existence of a newly defined operator are also proved.

\section{Acknowledgements}

Not applicable.

\section{Funding}

There is no funding for this work.

Availability of data and materials

Data sharing is not applicable to this paper as no datasets were generated or analyzed during the current study.

\section{Competing interests}

The authors declares that there is no conflict of interests regarding the publication of this paper.

\section{Authors' contributions}

All authors jointly worked on the results and they read and approved the final manuscript.

\section{Author details}

${ }^{1}$ Department of Mathematics, University of Sargodha, Sargodha, Pakistan. ${ }^{2}$ Department of Mathematics, Faculty of Science and Arts, Ordu University, Ordu, Turkey. ${ }^{3}$ Department of Mathematics, Shaheed Benazir Bhutto University, Sheringal, Pakistan.

\section{Publisher's Note}

Springer Nature remains neutral with regard to jurisdictional claims in published maps and institutional affiliations.

Received: 19 June 2019 Accepted: 4 October 2019 Published online: 17 October 2019

\section{References}

1. Agrawal, O.P., Baleanu, D.: Hamiltonian formulation and a direct numerical scheme for fractional optimal control problems. J. Vib. Control 13(9-10), 1269-1281 (2007)

2. Almeida, R.: Caputo-Hadamard fractional derivatives of variable order. Numer. Funct. Anal. Optim. 38(1), 1-19 (2017) 
3. Azam, M.-K., Farid, G., Rehman, M.-A.: Study of generalized type k-fractional derivatives. Adv. Differ. Equ. 2017, 249 (2017)

4. Diaz, R., Pariguan, E.: On hypergeometric functions and Pochhammer k-symbol. Divulg. Mat. 15, 179-192 (2007)

5. Dorrego, G.-A., Cerutti, R.-A.: The k-fractional Hilfer derivative. Int. J. Math. Anal. 7(11), 543-550 (2013)

6. Farid, G., Habullah, G.-M.: An extension of Hadamard fractional integral. Int. J. Math. Anal. 9(10), 471-482 (2015)

7. Gradshteyn, I.-S., Ryzhik, I.-M.: Table of Integrals, Series and Products, 7th edn. Elsevier, Amsterdam (2007)

8. Hadamard, J.: Essai sur lëtude des fonction données par leur dévelopment Taylor. J. Math. Pures Appl. 4(8), 101-186 (1892)

9. Jesus, I.-S., Machado, J.: Fractional control of heat diffusion system. Nonlinear Dyn. 54(3), 263-282 (2008)

10. Katugompola, U.-N.: New approach generalized fractional integral. Appl. Math. Comput. 218, 860-865 (2011)

11. Kilbas, A.-A., Sarivastva, H.-M., Trujillo, J.-J.: Theory and Applications of Fractional Differential Equations. North-Holland Mathematics Studies, vol. 204. Elsevier, Amsterdam (2006)

12. Leoni, G.: A First Course in Sobolev Spaces, 2nd edn. Am. Math. Soc., Providence (2009)

13. Qasim, M.-D., Furati, K.-M., Tatar, N.-E.: On a differential equation involving Hilfer-Hadamard fractional derivative. Abstr. Appl. Anal. 2012, Article ID 391062 (2012). https://doi.org/10.1155/2012/391062

14. Romero, L.-G., Luque, L.-L., Dorrego, G.-A., Cerutti, R.-A.: On the k-Riemann-Liouville fractional derivative. Int. J. Contemp. Math. Sci. 8(1), 41-51 (2013)

15. Samraiz, M., Afzal, M.-A., lqbal, S., Kashuri, A.: Opial-type inequalities for generalized integral operators with special kernels in fractional calculus. Commun. Math. Appl. 9(3), 421-431 (2018)

16. Samraiz, M., Iqbal, S., Pečarić, J.: Generalized integral inequalities for fractional calculus. Cog. Math. Stat. 5, 1426205 (2018)

\section{Submit your manuscript to a SpringerOpen ${ }^{\circ}$ journal and benefit from:}

- Convenient online submission

Rigorous peer review

Open access: articles freely available online

- High visibility within the field

- Retaining the copyright to your article

Submit your next manuscript at $\gg$ springeropen.com 